Revue d'histoire de l'Amérique française

REVUE D.HISTOIRE DE L'AMÉRIQUE FRANÇAISE

\title{
Les origines historiques du statut particulier
}

\section{Jacques-Yvan Morin}

Volume 20, numéro 1, juin 1966

URI : https://id.erudit.org/iderudit/302537ar

DOI : https://doi.org/10.7202/302537ar

Aller au sommaire du numéro

Éditeur(s)

Institut d'histoire de l'Amérique française

ISSN

0035-2357 (imprimé)

1492-1383 (numérique)

Découvrir la revue

Citer cet article

Morin, J.-Y. (1966). Les origines historiques du statut particulier. Revue

d'histoire de l'Amérique française, 20(1), 3-17. https://doi.org/10.7202/302537ar d'utilisation que vous pouvez consulter en ligne.

https://apropos.erudit.org/fr/usagers/politique-dutilisation/ 


\section{LES ORIGINES HISTORIQUES DU STATUT PARTICULIER *}

Le régime du statut particulier a été utilisé, sous de multiples formes et sous des noms divers, dans plusieurs états et empires du passé et au cours de la plupart des grands réaménagements politiques des Temps modernes, depuis la dissolution de l'Empire ottoman jusqu'à la constitution de l'Union soviétique, en passant par la dissociation de l'Empire britannique.

D'une manière générale, le statut particulier se présente comme une exception au principe de l'égalité ou de l'uniformité de régime des collectivités publiques qui composent un État. Une collectivité qui jouit d'un statut particulier possède, en sus des droits communs à toutes les autres collectivités, des droits ou des pouvoirs spéciaux et quelquefois une place à part dans les institutions du pays.

Le principe de l'égalité des collectivités publiques caractérise aujourd'hui la plupart des Etats, qu'ils soient unitaires ou fédératifs, du moins lorsqu'ils sont homogènes sur le plan ethnique. L'Union américaine constitue un exemple classique de fédération dont les Etats-membres sont égaux en ce qui concerne les compétences qui leur sont dévolues par la Constitution et la représentation à laquelle ils ont droit dans la Chambre haute. La Cour suprême des États-Unis n'a perdu aucune occasion d'affirmer que tout nouvel Etat entrait dans l'Union "sur le même pied" que les anciens et la doctrine de l'égalité constitutionnelle des Etats-membres est aujourd'hui acceptée comme un truisme par les juristes américains.

* Conférence prononcée le 30 avril 1966, au banquet de la réunion générale de l'Institut d'Histoire de l'Amérique française. 
Mon propos consiste à explorer quelques exemples de statuts particuliers tirés de l'Antiquité, à montrer comment les dirigeants britanniques ont eu recours à cette technique pour construire leur Empire et pour le transformer graduellement et pacifiquement en cette vague association d'États souverains qu'est le Commonwealth contemporain; enfin, à décrire le statut particulier embryonnaire que possède le Québec dans la Fédération canadienne et les chemins qui s'offrent à lui à la lumière de l'histoire.

\section{I}

\section{RÓLE HISTORIQUE DU STATUT PARTICULIER}

L'histoire révèle que toutes les grandes formations politiques et surtout les empires multinationaux ont utilisé d'une manière empirique ou de façon systématique les régimes particuliers.

L'Empire romain en fournit maints exemples. Sous la République, il existait déjà une grande diversité dans la situation juridique des peuples soumis et les libertés locales que conservaient les cités alliées ou vaincues variaient considérablement de l'une à l'autre. C'est ainsi que les provincix, territoires conquis dont une lex data définit la condition juridique et les obligations financières, ne jouissent pas des mêmes droits que les civitates fœederatæ, liées à Rome par un traité et qui conservent leurs institutions en échange des impôts qu'elles versent, ou les civitates liberæx, soumises à la domination romaine, mais exemptes d'impôts et conservant une certaine autonomie politique et administrative. À l'époque du Haut-Empire, ces inégalités tendirent à disparaître, grâce à l'organisation systématique du territoire en provinces impériales ou sénatoriales. Cependant, chaque province conservait, en partie du moins, ses institutions traditionnelles et son droit privé, qui subirent graduellement l'influence des institutions romaines. En outre, de nombreuses colonies bénéficiaient de situations privilégiées. L'Égypte, d'autre part, possédait un statut particulier caractérisé par l'absence de toute autonomie: elle faisait partie du patrimoine personnel de l'Empereur qui la gouvernait par l'entremise d'un préfet. 
Après la grande crise politique du III ${ }^{e}$ siècle, marquée par de nombreux mouvements séparatistes, Dioclétien centralisa fortement l'Empire: les provinces furent morcelées, regroupées en diocèses et soumises le plus possible à un régime uniforme. Par la suite, la tendance générale du Bas-Empire fut à l'élimination des particularismes, tant en droit public qu'en droit privé.

L'histoire institutionnelle de l'Empire romain, du II siècle av. J.C. au III ${ }^{e}$ siècle de l'ère chrétienne, est donc caractérisée par le grand nombre de statuts particuliers octroyés aux peuples soumis. Dans un monde si bigarré, il ne pouvait en être autrement: Rome découvrit que les institutions ne s'imposent guère par la force des armes et que la diversité des régimes n'est pas inconciliable avec l'intégration progressive des collectivités. Toute la phase ascendante de l'Empire est fondée sur cette politique, que nous retrouverons dans les empires des Temps modernes. Cependant, si le monde antique sut, exceptionnellement, trouver l'équilibre entre l'unité et les particularismes, la centralisation politique et économique qui caractérise l'Empire à compter $d u \mathrm{IV}^{e}$ siècle, empêche tout renouvellement des institutions locales à l'époque même où certaines provinces semblaient prendre conscience de leur identité nationale. Peut-être faut-il voir dans ce déséquilibre l'une des causes de la léthargie des populations et de la décadence finale de l'Empire.

\section{II}

\section{LES STATUTS PARTICULIERS DANS L'EMPIRE BRITANNIQUE}

Toute autre a été l'expérience de l'Empire britannique, vers laquelle nous nous tournons maintenant. Sans doute n'est-ce pas en vain que les jeunes gens, dans les grandes écoles d'Angleterre, furent pendant longtemps formés dans les livres de l'histoire romaine. Il est vrai que les hommes d'État britanniques ont eu sous les yeux le désastre que constitua leur premier empire (celui qui se révolta et devint les États-Unis) et la catastrophe humaine que fut le processus de dissociation de l'Empire ottoman. 
Leur empirisme sut tirer de ces exemples plus d'une leçon, comme je vais tenter de le démontrer.

\section{ECHEC DE LA PREMIÊRE POLITIQUE COLONIALE}

Mais revenons quelques instants à l'échec du premier Empire britannique. En Amérique, la variété des chartes et des concessions royales avait donné naissance à une multitude de statuts particuliers et chaque colonie était très jalouse de ses privilèges et de ses institutions qui correspondaient à ses origines - religieuses parfois - et à son niveau de développement. Les difficultés avec la métropole ne tardèrent pas à se faire jour lorsque les colonies réclamèrent une autonomie véritable par rapport à la Couronne et au Parlement britanniques.

Dès la fin du XVIIe siècle apparut le conflit qui allait aboutir à la guerre d'Indépendance. Certaines colonies se voyaient déjà comme les égales de la métropole, tandis que le Gouvernement de Guillaume III affirmait au contraire leur dépendance de la Couronne, bien qu'il n'ait pu supprimer les assemblées locales ni les statuts particuliers. Il en résulta une tentative de centralisation et une politique d'uniformité qui durèrent trois quarts de siècle. Le Gouvernement regroupa plusieurs colonies, réglementa leur commerce et attribua le monopole du transport maritime aux armateurs britanniques. Les intérêts du moment et la doctrine mercantiliste poussaient d'ailleurs la Grande-Bretagne vers ce contrôle toujours plus étroit des affaires coloniales, dont les premières étapes furent les Navigation Acts (1651, 1660, 1696) et dont l'aboutissement logique devait être le Declaratory Act de 1766, dans lequel il est réaffirmé, à la veille de la Révolution, que les colonies et plantations d'Amérique

have been, are, and of right ought to be, subordinate and dependant on the Imperial Crown and Parliament of Great Britain.

Aux anciens statuts particuliers, déjà si restreints, risquait de succéder l'uniformité impériale. Il n'est pas douteux que cette politique ait été l'une des causes essentielles de la déclaration d'indépendance des treize colonies. La dernière démarche du 
gouvernement de Londres en 1778, en pleine rébellion, le démontre: des émissaires du Parlement impérial vinrent proposer au Congrès l'abandon par la Grande-Bretagne de son pouvoir de taxation, sauf en ce qui concerne la réglementation du commerce, le revenu étant alors remis aux colonies; en outre, Sa Majesté et le Parlement renonçaient à modifier les constitutions des colonies en l'absence d'une requête émanant de l'Assemblée concernée et acceptaient de remplacer le Declaratory Act par une déclaration conjointe sur les relations constitutionnelles entre les diverses parties de l'Empire. En matière de commerce, le Parlement ne légiférait qu'après avoir pris l'avis des colonies; en ce qui concerne la défense, aucune armée permanente ne serait cantonnée dans les colonies sans leur consentement. Enfin, concession ultime, le Gouvernement britannique se disait prêt à accepter une représentation restreinte des colonies au Parlement impérial.

Cependant, ces concessions majeures, qui anticipaient l'avenir constitutionnel des autres colonies britanniques, venaient trop tard dans une Amérique mûre pour le self-government. Pour avoir refusé l'élargissement des statuts particuliers et abusé du pacte colonial, la Grande-Bretagne récolta l'indépendance des Etats-Unis. Cette dure expérience devait lui être très utile par la suite, particulièrement au Canada.

\section{LA NOUVELLE POLITIQUE COLONIALE : L'ACTE DE QUEBEC ET LE GOUVERNEMENT RESPONSABLE COMME STATUTS PARTICULIERS}

Après la conquête de la Nouvelle-France, le Gouvernement britannique songea à introduire dans la colonie le régime d'assemblée "de la manière prescrite et suivie dans les colonies et les provinces d'Amérique" et à créer des tribunaux civils pour juger les litiges "conformément autant que possible aux lois anglaises". Toutefois, on ne tarda pas à se rendre compte que les nouveaux sujets ne pouvaient être gouvernés à tous égards comme les autres habitants de l'Empire: les conseillers juridiques de la Couronne firent en effet rapport dès 1766 qu'il existait au Canada des causes de désordre, dont l'une était la crainte des Canadiens 
de voir abolir tous leurs usages et coutumes "avec la main rude du conquérant".

L'Acte de Québec (1774) établit donc pour la colonie du Canada un statut particulier limité mais véritable, en rétablissant, comme régime de droit civil, la loi en vigueur à l'époque des capitulations, disposition qui perpétuait notamment la tenure seigneuriale. En outre, le libre exercice de la religion catholique y était autorisé, le régime ecclésiastique romain était reconnu et les catholiques résidant dans la province n'étaient plus tenus de prêter le serment requis par le Test Act de 1673. Toutes ces dispositions constituaient d'importantes exceptions aux lois applicables dans l'ensemble de l'Empire, mais on doit signaler que la liberté de religion en faveur des sujets catholiques était déjà garantie par les capitulations de 1759 et 1760 , ainsi que par le Traité de Paris, du 10 février 1763. Dès les premières années qui suivirent la conquête se trouvèrent done réunis les éléments d'un régime particulier pour les sujets français du Canada. Cependant, ce n'était encore là qu'un statut spécial bien limité puisqu'aussi bien la population ne jouissait d'aucune autonomie politique, l'Acte de Québec ne lui accordant pas l'assemblée annoncée dans la Proclamation royale de 1763.

Cette chambre d'assemblée ne fut créée, en 1791, qu'après de longues hésitations de la part du Gouvernement britannique. Le régime représentatif ne tarda pas, en effet, à faire naître chez certains députés de la nouvelle province du Bas-Canada l'espoir d'une extension de son statut particulier et de son autonomie qui eût permis à la "nation canadienne" d'orienter librement son avenir. William Pitt n'avait-il pas déclaré, lors du débat sur l'Acte constitutionnel, que "dans le Bas-Canada, comme les résidents sont principalement des Canadiens, leur assemblée ... sera adaptée à leur coutume et à leurs idées particulières"? Aussi l'assemblée fut-elle amenée à revendiquer un statut que ne possédaient pas les autres colonies britanniques à l'époque: élection populaire du Conseil législatif, responsabilité du Conseil exécutif devant l'Assemblée, contrôle de tous les revenus. Comme le constatèrent en 1836 les Commissaires 
royaux chargés de faire enquête sur les griefs du Bas-Canada, ces prétentions n'étaient pas sans rappeler "la république américaine". Sans doute l'Assemblée se faisait-elle illusion sur les intentions réelles du Gouvernement impérial, car même les plus libéraux des Britanniques de l'époque, y compris Pitt et Grenville, espéraient que le self-government limité accordé aux Canadiens français leur enseignerait "que les lois anglaises sont les meilleures". Ces illusions contribuèrent à la révolte armée de 1837-38 qui entraîna la perte de l'Assemblée du Bas-Canada et, dans une certaine mesure, du statut particulier dont elle avait espéré doter le Canada français au sein de l'Empire.

Avec la Rébellion, qui avait agité également le Haut-Canada, la Grande-Bretagne se trouvait, une fois de plus, à la croisée des chemins: quelle politique constitutionnelle convenait-il d'appliquer dans ses colonies? Cependant, les leçons de la Révolution américaine rendaient les dirigeants plus souples et l'Angleterre s'était engagée depuis quelques années dans la voie de la réforme parlementaire. Le Gouvernement envoya au Canada l'un des membres les plus influents de l'aile libérale du parti au pouvoir, lord Durham, afin qu'il fît rapport sur la situation du pays. Dans son rapport, Durham opinait que la population française devait être assimilée pour son plus grand bien et dans l'intérêt de la métropole, entreprise qui supposait évidemment l'extinction graduelle du statut particulier embryonnaire accordé par l'Acte de Québec.

D'autre part, le noble Lord, qui avait été l'un des auteurs du premier Reform Bill en Grande-Bretagne (1831), estimait que l'une des causes de la rébellion avait été le conflit entre l'Assemblée et le Pouvoir exécutif, tant dans le Haut que dans le Bas-Canada. Il en conclut que la Couronne devait se soumettre aux conséquences nécessaires des institutions représentatives et consentir à l'établissement de la responsabilité ministérielle: "si elle doit faire fonctionner le gouvernement de concert avec un corps représentatif, il faut qu'elle y consente par l'intermédiaire de ceux en qui ce corps représentatif a confiance." Cependant, ce principe ne devait être appliqué "qu'à une assemblée déci- 
dément anglaise", d'où la nécessité d'unir les deux Canadas sous une même législature.

La recommandation d'étendre le gouvernement responsable à une colonie constituait une innovation remarquable pour l'époque. Aussi le cabinet britannique y vit-il d'abord un principe incompatible avec les relations qui doivent exister entre une métropole et ses colonies. Cependant, le gouvernement responsable fut accordé à la Nouvelle-Écosse (1846) et au Canada (1847) par le truchement des instructions adressées par le Colonial Office aux gouverneurs, dans lesquelles il leur était recommandé d'agir d'une façon générale sur l'avis d'un Conseil exécutif possédant la confiance de l'assemblée. Ces colonies se virent de la sorte attribuer un statut particulier au sein de l'Empire. Peu à peu, le gouvernement responsable devint la pierre angulaire de la nouvelle politique coloniale britannique; le statut particulier du Canaủa fut étendu aux autres territoires, chaque colonie bénéficiant des avantages déjà acquis par les plus anciennes, mais non sans tenir compte des circonstances particulières de chacune. On a pu écrire avec raison que le rapport Durham constituait "a Magna Carta of imperial history".

Toute l'évolution de l'Empire depuis la seconde moitié du $\mathrm{XIX}^{\mathrm{e}}$ siècle peut être décrite comme un ensemble de statuts particuliers évoluant à des rythmes différents vers l'égalité. Unitaire à l'origine et placé sous la suprématie sans limite de la métropole, le système juridique impérial, pour s'adapter à la diversité des situations géographiques, ethniques, économiques et sociales, a dû être diversifié et hiérarchisé. Cela est vrai des vieux dominions, dont le Canada a été le prototype constitutionnel, et pour les colonies qui ont accédé à l'autonomie et à l'indépendance après la seconde guerre mondiale.

\section{L'EXTENSION DU STATUT PARTICULIER CANADIEN AUX AUTRES DOMINIONS ET L'EGALITE DE STATUT}

Le statut du Canada, tant sur le plan interne que sur le plan externe, s'est sans cesse modifié depuis l'octroi du gouvernement responsable. Déjà avant 1845, lorsque la Grande-Bretagne passa 
au libre-échange, le gouvernement canadien, craignant les effets de la concurrence étrangère, revendiqua les pouvoirs nécessaires à l'érection d'un mur douanier; bientôt fut votée la loi impériale autorisant les colonies à adopter leur propre politique douanière et les Navigation Acts ne tardèrent pas à être abrogés (1849). En 1859, le Canada alla jusqu'à imposer des droits sur l'importation des marchandises britanniques; le Colonial Office dut s'incliner, malgré les protestations qui s'élevèrent aux Communes. De même, le Canada obtint de réglementer l'immigration, y compris celle des sujets britanniques et ce droit fut reconnu à tous les dominions vers 1901. Bien entendu, toute concession faite au Canada par le Colonial Office devait être étendue tôt ou tard aux autres colonies de peuplement. Ainsi, par un lent travail d'érosion, les compétences les plus importantes sur le plan de l'autonomie interne passèrent du Parlement impérial aux législatures coloniales, transformant sans cesse le statut particulier canadien en statut d'application générale et donnant à l'Empire l'allure d'une vague fédération.

Si nous tentons de résumer cette évolution, qui couvre la seconde moitié du $\mathrm{XIX}^{\mathrm{e}}$ siècle, nous pouvons y discerner deux phases principales. Dans un premier temps, au XIX $\mathrm{X}^{\mathrm{e}}$ siècle, l'effacement de la solidarité économique qui avait existé auparavant entre la métropole et les dominions ainsi que le particularisme croissant de territoires si différents et si éloignés les uns des autres n'offrent d'alternative qu'entre la sécession, voie qu'avaient choisie naguère les États-Unis, et l'élaboration de statuts spéciaux au fur et à mesure que le besoin s'en faisait sentir. L'empirisme des Britanniques et un certain libéralisme envers les dominions blancs, joints à l'absence de tout partage précis des compétences entre la métropole et ses colonies, favorisèrent l'évolution de l'Empire par la technique du statut particulier. Dans un second temps, l'apparition des conférences coloniales, à la fin du XIX $\mathrm{XI}^{\mathrm{e}}$ siècle, eut pour effet d'unifier l'attitude des colonies sur leurs problèmes communs et d'entraîner rapidement l'extension à tous les dominions des avantages obtenus par l'un d'entre eux; avec le $\mathrm{XX}^{\mathrm{e}}$ siècle, tout statut particulier 
tend donc à devenir général pour autant que les colonies de peuplement sont concernées.

L'évolution que nous venons de décrire n'est nulle part plus évidente qu'en matière de compétences internationales. Le Canada fut le premier à réclamer le privilège de participer à la négociation des accords commerciaux avec les autres pays, particulièrement après 1865 , mais trente ans plus tard, à la Conférence impériale de 1894, l'Australie et la Nouvelle-Zélande se firent accorder les mêmes droits. L'évolution fut plus lente en ce qui concerne les traités d'ordre politique et l'Empire conserva longtemps, à cet égard, un aspect unitaire prononcé, mais le Canada obtint d'être associé à la signature du Traité de Versailles et la même concession dut être faite aux autres dominions.

Arrivés à ce point, nous devons noter que le Canada ne posssèue plüs à compter de la première guerre moñudiale, de státut particulier par rapport aux autres dominions, en ce sens que tout avantage concédé par Londres au gouvernement canadien est étendu au même moment à l'Australie, à la NouvelleZélande et à l'Afrique du Sud. Les dominions aspirent tous maintenant à l'égalité de statut, non seulement entre eux, mais également à l'égard de la Grande-Bretagne. Cette égalité signifie avant tout autonomie et quasi-indépendance dans le cadre de l'Empire. Elle est consacrée d'une manière définitive, sauf à l'égard de l'Inde, lors de la Conférence de 1926, dans le rapport Balfour:

Ce sont, au sein de l'Empire britannique, des collectivités autonomes de statut égal; elles ne sont d'aucune manière subordonnées les unes aux autres... La tendance vers un statut égal était à la fois juste et inévitable. Les conditions géographiques et autres ont empêché d'atteindre ce but par voie fédérative.

Ainsi la technique de la diversité des statuts avait permis, en un demi siècle, de faire passer les colonies de peuplement au rang d'Etats pleinement autonomes. Pour cela, il avait fallu éviter toute constitution impériale précise, tout fédéralisme rigide (on sait comment Laurier s'opposa au projet de fédération im- 
périale) et tout mode d'amendement constitutionnel fixe. C'est sans doute pour cette raison que maints juristes ont renoncé à donner de l'Empire et du Commonwealth une définition exacte. Selon les mots de lord Balfour lui-même, "l'Empire défie toute classification et n'a aucune ressemblance véritable avec les organisations existantes non plus qu'avec aucune de celles qui ont existé dans le passé."

\section{III}

\section{LE STATUT PARTICULIER DU QUÉBEC}

Le Québec possède depuis longtemps, au sein de l'Empire britannique, un statut spécial qui s'est transformé en 1867 en statut particulier dans la Fédération canadienne. Déjà en 1774, l'Acte de Québec lui avait accordé des droits que nulle autre colonie ne pouvait revendiquer, en rétablissant le libre exercice de la religion catholique et le droit civil. Ce statut fut maintenu dans les faits à l'époque de l'Union législative, grâce à l'acharnement avec lequel les Bas-Canadiens s'ingénièrent à instaurer une sorte de dualisme pratique dont la manifestation la plus typique fut le système de la double majorité. C'est ainsi que la province, en 1867, échappa aux dispositions de l'A.A.N.B. qui favorisaient l'uniformité des lois relatives à la propriété et au droit civil dans les autres provinces.

\section{UN STATUT PARTICULIER BIEN CIRCONSCRIT}

En outre, il est prévu dans la Constitution que les juges des tribunaux québecois doivent être choisis parmi les membres du Barreau de la province. Toutefois, on doit noter que le Parlement fédéral devenait compétent à l'égard d'un certain nombre de sujets qui relevaient traditionnellement du droit civil, comme le mariage et la faillite, par exemple; les dispositions de l'article 91 restreignent donc le régime spécial défini par l'Acte de Québec.

Le premier fait qui frappe l'observateur, si l'on se place dans une perspective historique, c'est que l'A.A.N.B. figeait le statut particulier du Québec et empêchait théoriquement tout 
développement ultérieur de ce statut. En réalité, ce qui prime dans l'A.A.N.B., c'est l'égalité de statut: le Québec est sur le même pied que les autres provinces, sauf à l'égard du droit privé et de quelques autres dispositions dont il sera question à l'instant. Alors que le Canada et les autres dominions obtenaient l'égalité de statut presque au terme de leur évolution constitutionnelle, le Québec n'y accède qu'à un niveau inférieur: celui de l'État-membre d'une fédération qui, au demeurant, devait être hautement centralisée. En somme, l'entrée dans la Fédération canadienne mettait fin au développement normal du statut particulier du Québec dans le sens d'une autonomie toujours croissante. La Grande-Bretagne n'a pas voulu accorder au Québec, pour des raisons d'ordre politique et économique, le traitement qu'elle a accordé à ses dominions.

Il existe un autre aspect du statut particulier du Québec,

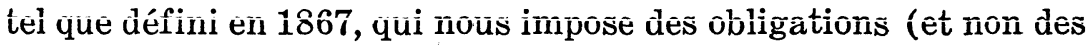
droits) auxquelles ne sont pas astreintes les autres provinces. C'est ainsi que la représentation anglophone à l'Assemblée législative était assurée en "gelant" les limites de douze circonscriptions électorales où il existait à l'époque une forte majorité anglaise. De même, on pensait protéger cette représentation dans le Sénat fédéral et au Conseil législatif provincial en fixant d'avance les limites des 24 circonscriptions du Québec. Enfin, la province est la seule où l'usage des deux langues officielles fut consacré par l'A.A.N.B., tant dans les débats de la Législature que devant les tribunaux. C'est là, s'il m'est permis de m'exprimer ainsi, un statut particulier "négatif", en ce sens qu'il est inférieur à celui des autres provinces. Il est vrai qu'on a imposé un statut analogue au Manitoba, lors de la création de cette province, pour la protection de la langue française, mais chacun sait comment elle s'en débarrassa, une vingtaine d'années plus tard, sans autre forme de procès.

\section{RENOUER LE FIL DE L'HISTOIRE}

Cependant, depuis quelques années se fait jour au Québec l'opinion selon laquelle le Québec doit posséder un véritable statut particulier qui lui permettrait d'obtenir toute l'autonomie 
dont il a besoin comme "foyer national" du Canada français. En 1956, le rapport de la Commission Tremblay déclarait que le Québec constitue notre "milieu politique fondamental" parce que, comme communauté humaine, il n'est assimilable à aucune autre "ni par les origines, ni par la religion, ni par la culture, ni par l'histoire de la grande majorité de sa population, ni en conséquence par la plupart de ses institutions juridiques et sociales". C'est pourquoi la Commission en vient à la conclusion que le Canada français a besoin d'un territoire où il puisse librement s'exprimer et bâtir ses propres structures institutionnelles. Pour en arriver là, les commissaires faisaient appel surtout à des moyens fiscaux, sans réclamer de grands changements constitutionnels, si ce n'est à l'égard de la Cour constitutionnelle et du mode d'amendement de la Constitution. Peut-être espéraiton que toutes les provinces imitent le Québec, du moins en matière fiscale.

Il a été démontré au cours des dix dernières années que la philosophie du fédéralisme qui se dégage du rapport Tremblay n'est point partagée par le Canada anglais. Sauf de rares exceptions, on ne retrouve chez les provinces anglophones ni le profond souci d'autonomie du Québec, ni la tendance à chercher dans le cadre étatique provincial la garantie de l'épanouissement culturel d'un groupe ethnique, ni même l'idée d'un Canada fondé en permanence sur la coexistence de deux peuples distincts. Au contraire, comme l'indique le récent rapport préliminaire de la Commission fédérale d'enquête sur le bilinguisme et la dualité de culture, présidée par MM. Laurendeau et Dunton, la plupart des Canadiens dont l'ascendance n'est pas française semblent se faire du Canada une conception essentiellement unitaire. Au sein de la "nation canadienne", c'est la majorité qui doit gouverner, a-t-on répété à maintes reprises devant les commissaires. Et le rapport d'ajouter: "Très souvent aussi, on a formulé la règle de la majorité simple en fonction des provinces, le Québec étant alors l'une des dix unités égales qui constituent le pays."

Le heurt de ces deux conceptions contraires du fédéralisme et de l'État constitue très certainement l'une des causes pro- 
fondes de la crise que traversent à l'heure actuelle les institutions canadiennes. $\grave{A}$ compter du moment où le Canada anglais ripostait à la thèse de la "mission particulière" du Québec par l'affirmation de l'égalité de toutes les provinces, les Canadiens français, dans un contexte international nouveau qui favorise le droit de libre disposition des peuples, ne pouvaient que renouer le fil rompu de l'histoire et reprendre le développement du statut particulier du Québec là où les évolutions de 1840 et de 1867 l'avaient laissé.

Les défenseurs d'un statut particulier moderne pour le Québec veulent élargir cette notion et y faire entrer un certain nombre de compétences qui relèvent à l'heure actuelle du Parlement fédéral; ils proposent également de redéfinir le mode de participation du Québec au fonctionnement des organes centraux de l'État fédéral. J'estime, pour ma part, qu'une solution de ce type s'impose, si l'on veut vraiment faire en sorte "que la Confédération canadienne se développe d'après le principe de l'égalité entre les deux peuples qui l'ont fondée".

Le statut particulier du Québec doit être consacré tôt ou tard par le droit constitutionnel écrit. Certes, cette idée ne manque pas d'éveiller de fortes résistances au Canada anglais, mais telle est pourtant la condition d'un juste équilibre entre les deux nations canadiennes. Un observateur de l'extérieur, le journaliste Claude Julien, écrivait récemment à ce propos que les réalistes devront reconnaître que le Québec possède de fait une situation particulière méritant d'être consacrée par un statut particulier. Le refus d'un tel aménagement, ajoute-t-il, précipiterait l'éclatement de la Confédération et cet éclatement serait la fâcheuse conséquence non pas tant du séparatisme québecois que de l'incompréhension des anglophones, qui en porteraient l'entière responsabilité.

Nous sommes donc à la croisée des chemins et c'est sur cette vision que je veux terminer cet exposé. J'ai parlé du passé, 
comme il sied devant un institut d'histoire, mais il est clair que la lumière d'un passé toujours mieux compris perce les ténèbres de l'avenir. Ce fut l'expérience du passé, c'est-à-dire de l'homme, qui fit d'un Tocqueville, par exemple, un véritable prophète politique. N'est-ce pas lui qui a montré, dès 1840, comment les peuples démocratiques reçoivent spontanément les idées simples et générales et ne s'accommodent guère des "systèmes compliqués". "L’idée des pouvoirs secondaires, écrit-il, est naturellement absente de l'esprit des hommes dans les siècles d'égalité ...; tandis qu'ils conçoivent, pour ainsi dire sans y penser, l'idée d'un pouvoir unique et central qui mène tous les citoyens par lui-même... Après l'idée d'un pouvoir unique et central, celle qui se présente le plus spontanément à l'esprit des hommes, dans les siècles d'égalité, c'est l'idée d'une législation uniforme."

Aujourd'hui, le Canada français est comme un bolide lancé à folle allure et en pleine nuit sur les chemins de l'avenir, qui sont pleins d'obstacles et de virages inattendus. C'est pourquoi il faut regarder en avant, de manière à éviter d'entrer dans l'avenir à reculons, comme disait Valéry. Mais nos phares doivent porter toujours plus loin et permettre de voir plus large, de manière que l'avenir ne se présente point comme une fatalité inexorable et que le changement, voulu et construit dans toute la mesure du possible, cesse d'effrayer. Or l'un de nos phares, c'est la connaissance historique, qui nous révèle l'enchaînement des causes et des effets. C'est en grande partie grâce à elle que le Canada français se reconnaîtra et demeurera lui-même dans un monde en transformation.

JACQUES-YVAN MORIN, professeur de droit constitutionnel à l'Université de Montréal. 